\title{
A new reduced control design based on the theory of wave domain control
}

\author{
Quan Wang, A.Y.T. Leung*, Dajun Wang \\ Department of Mechanics, Peking University, P.R. China \\ *Department of Civil and Structural Engineering, \\ University of Hong Kong, Hong Kong
}

\begin{abstract}
The work in this paper is based on the theory in references[1-5]. The main idea is to establish a transformation, which changes the original system into an image system, in which the control force is designed in the context of wave domain control and wave control, so that the number of degrees of freedom in the undisturbed state of the image system can be reduced. The design of control in the original system can be derived by inverse transformation. This method, compared with that in reference[2], is more general and is easy to apply.
\end{abstract}

\section{INTRODUCTION}

In structural analysis, the number of degrees of freedom is large when the structure is discretized by, such as, finite element method. The number of degrees of freedom of a large space structure after discretization may be hundred, thousands, or even more. It is adventageous to reduce the number of degrees of freedom in a control design based on the following reasons. The complexity of computation increases exponentially with the number of degrees of freedom, as evidence in the higher order Ricatti equation. The number and placements of actuators and sensors are not free, especially in large space structures. Otherwise, the primary behavior of the space structure may be changed, and the design on the original structure will be invalid. On the other hand, the orders of control equation are also suited for fewer actuators and sensors. For example, in the IMSC (Independent Modal Space Control), only $n$ number of modes can be controlled for $n$ actuators and sensors. The computation of large order matrices increases the round-off errors, in particular, in the higher modes of structure. And, finally the hardware may encounter difficulties for the large number of degrees of freedom. For example, the capacity of the on orbit computer needs to be considered.

Two kinds of approaches for the design of the reduced order control are of interest. ${ }^{[t-1,]}$

One is the open-loop reduced order, where the order of the mathematical model of the original system is directly reduced and the usual design of system is obtained on the basis of the reduced order model. The other is close-loop reduced order, that is, the order of the controller, that is derived on the basis of the original system, is directly reduced.

0-7803-2559-1/95 \$4.00 (C) 1995 IEEE
This paper is based on the theory of wave domain control and wave control presented in [1-5]. A reduced order control design is given in reference[3-4]. The main idea is that when the initial disturbance is given, perhaps in the full spatial domain, the state that is reduced is almost zero with the control force obtained by the theory of structural wave domain control introduced in section II. It is very difficult in applications when the endurance of structure and the performance of controller are to be considered. Furthermore, the design of this method is affected by the degrees of controllability of the structural wave domain control, which can not be given freely.

The main idea of our work is to establish a transformation, with which the original system is changed to an image system. The initial disturbance in the image system, which is transformed from the initial displacement or other state variable, must be spatial domain defined so that the theory on structural wave domain control can be used to design the control force, that enables some other states of the image system remain undisturbed and reducible. The procedure of reducing order is in the image system. After the design of reduced order control in the image system, the control design and the state response in the original system can be derived by inverse transformation. When the transformation is non-unique, a 'better' image system can be found, in which the number of degrees of controllability of wave domain control is large, so the reduced orders can be high, and the control design is easier to realize. A new path is opened in the research of structural design of reduced order control.

\section{STRUCTURAL WAVE DOMAIN CONTROL[1-5]}

We summarize our results in the field of wave control and the degree of controllability for later discussion. The state equation of a system is expressed as:

$$
\dot{\mathrm{x}}=\mathrm{Ax}+\mathrm{Bu}
$$

where $A$ and $B$ are the matrices of system and control respectively, $\mathrm{x}$ and $\mathrm{u}$ are vectors of state and control respectively, namely

$$
A \in R^{n \times n}, B \in R^{n \times r}, x \in R^{n}, u \in R^{r}
$$

In classical theory, that system (1) is state controllable means: for any initial state $x_{0}$ and time $t$, the control force $u(\tau)(\tau \in(0, t))$ can be derived, so that the state can be controlled at all time $t$. 
It is shown that the definition does not characterize the dynamic features that the response can be zero at any time in a spatial domain when appling the control force. In order to give further analyses, some symbols are introduced at first. $A(i, j)$ is the matrix with its entries consisting of the first $i$ rows and the first $j$ columns of matrix $A$ and $A(i, j)$ is that with the last $i$ rows and the first $j$ columns of matrix $A$. In a similar way, $B(i, j)$ and $B(i, j)$ can be defined. $x(t, i)$ is a vector with the first $i$ elements of vector $x$ and $x(t, i)$ is that with the last $i$ elements of $x . P_{n \times j}$ is a matrix, whose column vectors are that of some $\mathrm{j}$ column vectors of unit matrix $I_{n \times n}$. On the other hand, $\widetilde{P}_{n \times j}$ is a matrix, whose column vectors are that of the other $n-j$ column vectors of $I_{n \times n}$

$$
\begin{array}{cc}
B_{11}=B(n-i, r) P, & B_{12}=B(n-i, r) \bar{P} \\
\mathrm{~B}_{21}=\mathrm{B}(\mathrm{P} \underline{\mathrm{i}}, \mathrm{r}), & B_{22}=\mathrm{B}(\mathrm{i}, \mathrm{r}) \bar{P} \\
A_{11}=A(n-i, n-i), & A_{21}=\mathrm{A}(\mathrm{i}, \mathrm{n}-\mathrm{i})
\end{array}
$$

The matrix $\mathrm{P}$ must satisfy the following equation:

$$
\mathrm{R}(\mathrm{B}(\mathrm{i}, \mathrm{r}) \mathrm{P})=\mathrm{R}(\mathrm{B}(\mathrm{i}, \mathrm{r}))
$$

where $R($.) means the spanning space of the corresponding matrix, and $B_{21}{ }^{+}$is the pseudo-inverse of $\mathrm{B}_{21}$.

$$
\text { Let }
$$$$
\bar{B}=B_{12}-B_{11} B_{21}{ }^{+} B_{22}, \bar{A}=A_{11}-B_{11} B_{21}{ }^{+} A_{21}
$$$$
\bar{B}=B_{21}-B_{11} B_{21}{ }^{+} B_{22}, \bar{A}=A_{11}-B_{11} B_{21}{ }^{+} A_{21}
$$

From wave dynamics, we give the following definitions and criteria.

Definition 1: Eq.(1) is controllable, if $\exists i(i$ is an integer less then $\mathrm{n}), \forall x(0, n-i), \exists u(\tau)(\tau>0)$, which result in $x(t, i)=0$ for all $t>0$.

Definition 2: The degree of controllability of Eq.(1) is defined as the maximum of the integer $i$ in Definition 1. The criteria of controllability are given below.

Criterion 1: The degree of controllability of Eq.(1) is i, iff

$\mathrm{R}(\mathrm{B}(\mathrm{i}, r)) \supset \mathrm{R}(\mathrm{A}(\mathrm{i}, \mathrm{n}-\mathrm{i}))$, i.e.

$\operatorname{rank}(\mathrm{B}(\mathrm{i}, \mathrm{r}))=\operatorname{rank}(\mathrm{B}(\mathrm{i}, \mathrm{r}), \mathrm{A}(\mathrm{i}, \mathrm{n}-\mathrm{i}))$

The physical significance of the above definitions and criteria are that they are used to testify whether a given spatial domain can be disturbed with the applied control force, when a disturbance in a spatial domain is given. A simple proof of the criterion and numerical simulation with the model of spring-mass system are given in reference[1] and Appendix A.

Definition $3 \mathrm{~A}$ structure is wave controllable means, $\exists i(i<n), \forall x(0, n-i), \forall t>0$,

$\exists u(\tau)(\tau \in(0, t))$,

which result in

$x\left(t^{\prime}, \mathrm{i}\right)=0 .\left(t^{\prime} \geq 0\right)$ and $x(t, n-i)=0$.

Next, a sufficient condition for structural wave control is given.

Criterion 2 A structure is wave controllable, if

a. $\exists i(i<n), \mathrm{R}(\mathrm{B}(\mathrm{i}, \mathrm{r})) \supset \mathrm{R}(\mathrm{A}(\mathrm{i}, \mathrm{n}-\mathrm{i}))$ b. $\exists P$, which makes

rank $\left(\bar{B}, \bar{A} \bar{B}, \ldots, \bar{A}^{(n-i-1)} \bar{B}\right)=n-i$

The physical significance of above definitions and criteria is that they can be used to testify whether the structure can be controlled to zero state, meanwhile the given spatial domain can not be disturbed with the applied control force, when a disturbance

in a spatial domain is given. The proof of criterion 2 and some numerical simulations with the models of springmass-system, string and Euler beam are given in reference[2].

\section{THE DESIGN OF A REDUCED ORDER CONTROL}

Consideration the transformation of system (1),

$$
\begin{aligned}
& \{\mathrm{x}\}=\left[\begin{array}{ll}
\varphi_{11} & \varphi_{12} \\
\varphi_{21} & \varphi_{22}
\end{array}\right]\left\{\begin{array}{c}
\mathrm{v}_{1} \\
\mathrm{v}_{2}
\end{array}\right\}=[\varphi]\{\mathrm{v}\} \\
& v_{1} \in R^{l}, v_{2} \in R^{r-l}, \varphi_{11} \in R^{(\times l}, \\
& \varphi_{12} \in R^{l \times(r-l)}, \varphi_{21} \in R^{(r-l) \times l}, \text { The } \\
& \varphi_{22} \in R^{(r-l) \times(r-l)}
\end{aligned}
$$

transformation must satisfy the following conditions:

1. $[\varphi]$ can be inversed.

2. $\{\mathrm{x}\}_{0}=[\varphi]\{\mathrm{v}\}_{0}$

$$
\text { 3. }\left\{v_{2}\right\}_{0}=\left[\begin{array}{llll}
0, & 0, & \ldots, & 0
\end{array}\right]^{T} \text {, }
$$

$\{x\}_{0}$ and $\{v\}_{0}$

are initial conditions in different system respectively.

Substituting Eq.(2) into Eq.(1), one gets

$$
[\varphi]\{\ddot{\mathrm{v}}\}=\mathrm{A}[\varphi]\{\mathrm{v}\}+\mathrm{Bu}
$$

Multiplying $\varphi^{-1}$ on the both sides of the above equation, we have the following system

$$
\ddot{\mathrm{v}}=\varphi^{-1} \mathrm{~A} \varphi \mathrm{v}+\varphi^{-1} \mathrm{Bu}=\overline{\mathrm{A}} \mathrm{v}+\overline{\mathrm{B}} \mathrm{u}
$$

which is called the image system.

Now, the control force is designed in the image system by the theory of structural control[2], which makes the disturbance to be absorbed on the determined spatial domains, so that they are not disturbed and to be reduced. Then the control design of the original system can be derived by the transformation equation (2). Let the degree of controllability of Eq.(4) be $i$, according to criterion 1, Eq.(4) is rewritten as

$$
\begin{aligned}
\left\{\begin{array}{c}
\dot{v}_{3} \\
\dot{v}_{4}
\end{array}\right\} & =\left[\begin{array}{ll}
A_{11} & A_{12} \\
A_{21} & A_{22}
\end{array}\right]\left\{\begin{array}{l}
v_{3} \\
v_{4}
\end{array}\right\} \\
& +\left[\begin{array}{ll}
B_{11} & B_{12} \\
B_{21} & B_{22}
\end{array}\right]\left\{\begin{array}{l}
u_{1} \\
u_{2}
\end{array}\right\}
\end{aligned}
$$

where

$v_{3} \in R^{(n-i)}, v_{4} \in R^{i}, \mathrm{u}_{1} \in R^{(r-i)}, u_{2} \in R^{i} A_{m j}$ and $B_{m j} \quad(m=1,2, j=1,2)$ are corresponding matrices. Assume Eq.(5) be wave controllable, that is, criterion 2 is satisfied. So state $v_{4}$ is reduced, and Eq.(5) become 


$$
\begin{aligned}
& A_{21} v_{3}+B_{21} u_{1}+B_{22} u_{2}=0 \\
& \dot{\mathrm{v}}_{3}=\mathrm{A}_{11} \mathrm{v}_{3}+\mathrm{B}_{11} \mathrm{u}_{1}+\mathrm{B}_{12} \mathrm{u}_{2}
\end{aligned}
$$

that is the reduced order model, on which the control force is designed. From transformation Eq.(2), we have

So

$$
\{\mathrm{x}\}=[\varphi]\{\mathrm{v}\}=\left[\begin{array}{ll}
\varphi_{11} & \varphi_{12} \\
\varphi_{21} & \varphi_{22}
\end{array}\right]\left\{\begin{array}{l}
\mathrm{v}_{3} \\
\mathrm{v}_{4}
\end{array}\right\}
$$

$$
\{\mathrm{x}\}=\left\{\begin{array}{c}
\mathrm{x}_{3} \\
\mathrm{x}_{4}
\end{array}\right\}=\left\{\begin{array}{l}
\varphi_{11} \mathrm{v}_{3} \\
\varphi_{21} \mathrm{v}_{3}
\end{array}\right\}
$$

which is the state response of the original system. The procedure of obtaining Eq.(5),(6),(7), and the design of control force have been all discussed in reference[1-5].

First, we take the model of spring-mass system expressed in Fig.1 to illustrate our idea.

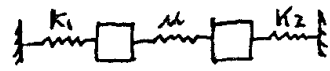

Fig. 1

The control equation of the system is

$$
\{\ddot{\mathrm{x}}\}+[\mathrm{K}]\{\mathrm{x}\}=[\mathrm{B}]\{\mathrm{u}\}
$$

where

$$
\begin{gathered}
{[K]=\left[\begin{array}{cc}
k_{1}+\mu & -\mu \\
-\mu & k_{2}+\mu
\end{array}\right] \quad[B]=\left[\begin{array}{ll}
b_{11} & b_{12} \\
b_{21} & b_{22}
\end{array}\right]} \\
\{u\}=\left\{\begin{array}{l}
u_{1} \\
u_{2}
\end{array}\right\}
\end{gathered}
$$

Let the initial displacement be

$$
\{x\}_{0}=\left[\begin{array}{ll}
1 & -1
\end{array}\right]^{\mathrm{T}}
$$

The transformation matrix and its inverse form are

$$
\varphi=\left[\begin{array}{cc}
1 & 0 \\
-1 & 1
\end{array}\right], \quad \varphi^{-1}=\left[\begin{array}{cc}
1 & 0 \\
+1 & 1
\end{array}\right]
$$

The transformation is

$$
x=\varphi v
$$

Substituting initial condition (9) into the above equation, the initial disturbance in image system is derived as

$$
\{v\}_{0}=\left[\begin{array}{ll}
1 & 0
\end{array}\right]^{\mathrm{T}}
$$

The image system is

$$
\ddot{\mathrm{v}}+\varphi^{-1} \mathrm{~K} \varphi \mathrm{v}=\varphi^{-1} \mathrm{Bu}
$$

That is

$$
\ddot{v}+\bar{K} v=\bar{B} u
$$

where

$$
\begin{aligned}
{[\bar{K}] } & =\left[\begin{array}{ll}
k_{1}+2 \mu & -\mu \\
k_{1}-k_{2} & k_{2}
\end{array}\right] \\
{[\bar{B}] } & =\left[\begin{array}{cc}
b_{11} & b_{12} \\
b_{11}+b_{21} & b_{12}+b_{22}
\end{array}\right]
\end{aligned}
$$

Let $\quad b_{11}+b_{12} \neq 0$

and

$b_{11}\left(b_{12}+b_{22}\right) \neq\left(b_{11}+b_{21}\right) b_{12}$, to satisfy criterion

2 . So the degree of controllability of system (12) is 1 , and the system is wave controllable. Then state $v_{2}$ is reduced, the reduced model is

$$
\begin{aligned}
\ddot{v}_{1}+\left(\mathrm{k}_{1}+2 \mu\right) \mathrm{v}_{1} & =\mathrm{b}_{11} \mathrm{u}_{1}+\mathrm{b}_{12} \mathrm{u}_{2} \\
\left(k_{\mathrm{1}}-k_{2}\right) v_{1} & =\left(b_{11}+b_{21}\right) u_{1} \\
& +\left(b_{12}+b_{22}\right) u_{2}
\end{aligned}
$$

From Eq.(14), we have

$$
\begin{aligned}
u_{1} & =\frac{1}{b_{11}+b_{21}}\left[\left(b_{12}+b_{22}\right) u_{2}\right. \\
& \left.-\left(k_{1}-k_{2}\right) v_{1}\right]
\end{aligned}
$$

Substituting Eq.(15) into Eq.(13), one gets

$$
\ddot{\mathrm{v}}_{1}+\left(\mathrm{k}_{1}+2 \mu\right) \mathrm{v}_{1}=\mathrm{c}_{1} \mathrm{u}_{2}+\mathrm{c}_{2} \mathrm{v}_{1}
$$

where

$$
\begin{aligned}
c_{1} & =\frac{1}{b_{11}+b_{21}}\left[\left(b_{12}+b_{22}\right) b_{11}\right. \\
& \left.+\left(b_{11}+b_{21}\right) b_{12}\right] \\
c_{2} & =\frac{1}{b_{11}+b_{21}}\left(k_{2}-k_{1}\right)
\end{aligned}
$$

For the convenience of illustration, let

$$
\begin{aligned}
& k_{1}=k_{2}=k \quad b_{12}=-b_{22}=1 \\
& b_{11}=b_{21}=1
\end{aligned}
$$

So $u_{1}=0$.

Eq.(16) changes to

$$
\ddot{\mathrm{v}}_{1}+\left(\mathrm{k}_{1}+2 \mu\right) \mathrm{v}_{1}=\mathrm{u}_{2}
$$

The control force $u_{2}$ is designed based on Eq.(17), and the state $v_{1}$ is controlled to a determined one. From transformation (10), one has

$$
\{\mathrm{x}\}=\left\{\begin{array}{l}
\mathrm{x}_{1} \\
\mathrm{x}_{2}
\end{array}\right\}=[\varphi]\{\mathrm{v}\}=\left[\begin{array}{cc}
1 & 0 \\
-1 & 1
\end{array}\right]\left\{\begin{array}{c}
\mathrm{v}_{1} \\
0
\end{array}\right\}
$$

That is, $x_{1}=v_{1} x_{2}=-v_{1}$, which are the response after control in the original system. Next, we will put the design of control force derived from the image system into the original system to verify if the response is the same as expressed in Eq.(18).

The original equation is

$$
\begin{aligned}
& \ddot{x}_{1}+\left(k_{1}+\mu\right) x_{1}-\mu x_{2}= \\
& b_{11} u_{1}+b_{12} u_{2}=u_{1}+u_{2} \\
& \ddot{x}_{2}+\left(k_{2}+\mu\right) x_{2}-\mu x_{1}= \\
& b_{21} u_{1}+b_{22} u_{2}=u_{1}-u_{2}
\end{aligned}
$$

Summing both sides of above equations, one gets

$$
\left(\ddot{\mathrm{x}}_{1}+\ddot{\mathrm{x}}_{2}\right)+\mathrm{k}\left(\mathrm{x}_{1}+\mathrm{x}_{2}\right)=2 \mathrm{u}_{1}=0
$$

For the initial condition of state $x_{1}+x_{2}$ is 0 , we obtain

$$
x_{1}=-x_{2}
$$

Introducing Eq.(21) into Eq.(19), we have

$$
\ddot{x}_{1}+\left(k_{1}+2 \mu\right) x_{1}=u_{2}
$$

Compared with Eq.(17), we know that,

$$
x_{1}=v_{1}
$$




$$
x_{1}=v_{1} \quad \mathrm{x}_{2}=-v_{1}
$$

which is the same as that in Eq.(18).

Now, consider the Euler beam as model to illustrate our theory. The equation of an Euler beam is

$$
\rho A \frac{\partial^{2} y}{\partial t^{2}}+E I \frac{\partial^{4} y}{\partial x^{4}}=f(x, t)
$$

Without losing generality, let the material parameter $\frac{E I}{\rho A}=1$, the length of beam $l=10$.

The model of differential discretization of Euler beam is given as

$$
\{\ddot{\mathrm{y}}\}+[\mathrm{K}]\{\mathrm{y}\}=\{\mathrm{f}\}
$$

where

$$
[\mathrm{K}]=\frac{\mathrm{EI}}{\rho \mathrm{A \Delta \textrm {x } ^ { 4 }}}\left[\begin{array}{ccccccc}
5 & -4 & 1 & & & & \\
-4 & 6 & -4 & 1 & & & \\
1 & -4 & 6 & -4 & 1 & & \\
& & & \ddots & & & \\
& & & -4 & 6 & -4 & 1 \\
& & & & -4 & 6 & -4 \\
& & & & 1 & -4 & 5
\end{array}\right]
$$

The initial displacement in the original system is shown in Fig.2, and the initial disturbance in the image system is shown in Fig. 3 when a transformation matrix $\varphi$ is given. The vertical axis expresses the response of the displacement of structure, and the horizontal axis expresses the length of structure. Let the criteria of wave domain and wave control are satisfied in the image systems. The design of control force and the displacement response are shown in Fig.4-5 by the classical method of IMSC (Independent modal space control). After inverse transformation, we get the design of control force and the displacement in the original system, which are shown in Fig.6-7. For Fig.4-Fig.7, the $x$ axis expresses the length of structure, the $y$ axis expresses the time corresponding to the response, and the $\mathrm{z}$ axis expresses the response of the displacement of structure. The time history is 5 seconds.

\section{CONCLUSIONS AND REMARKS}

A new idea of structural reduced order control is introduced in this paper, which is based on the concepts and criteria of structural wave domain control and wave control. Compared with the existed works on the design of structural reduced order, our work has the following characteristics. (1.) All the design of control is derived in one system, named image system that has strong physical significance. So the theory of wave domain control and wave control may be applied in the design of control. (2.) The numbers of the reduced order of this method can be larger depending on the selection of image systems, unlike the method in references[3-4], where the reduced orders are affected by the degrees of controllability. (3.) It can be seen that, from the procedure of this method, important physical information is captured. The reduced state in the image system is undisturbed with the control force applied, not like other researches in this field that the state is reduced according to some indexes, such as the degree of state controllability.

There are still many further works. That which image system is 'better' is still unanswered due to the large selections of image system with the initial state in the original system and the initial disturbance given in image system. All the above researches rely on the further studies of the theory and application of wave domain control and wave control.

\section{ACKNOWLEDGEMENT}

The work is supported by the National Science Foundation of China and Research Grant Council of Hong Kong.

\section{Appendix A}

Criterion 1: The degree of controllability of Eq.(1) is $\mathrm{i}$, iff $\mathrm{R}(\mathrm{B}(\mathrm{i}, r)) \supset \mathrm{R}(\mathrm{A}(\mathrm{i}, \mathrm{n}-\mathrm{i}))$, i.e.

$\operatorname{rank}(\mathrm{B}(\mathrm{i}, \mathrm{r}))=\operatorname{rank}(\mathrm{B}(\mathrm{i}, \mathrm{r}), \mathrm{A}(\mathrm{i}, \mathrm{n}-\mathrm{i}))$

A simple explanation of criterion 1 is given.

If the degree of controllability of Eq.(1) is $i$, we have $A(i, n-i) \times(t, n-i)=B(i, r) u(t)$

which means that it requires there exists $u(t)$ in order that Eq.(a) should be satisfied for any given $x(t, n-i)$. The condition of such problem is

$$
R(B(i, r)) \supset R(A(i, n-i))
$$

Now we give an example to illustrate the significance and application of the definition and the criterion in design of control force.

The equation is (not losing the generality, $m_{1}=m_{2}=1$ )

$$
\begin{aligned}
& \ddot{\mathrm{x}}_{1}+\left(\mathrm{k}_{1}+\mathrm{m}\right) \mathrm{x}_{1}-\mathrm{mx}_{2}=\mathrm{f}_{1}(\mathrm{t}) \\
& \ddot{\mathrm{x}}_{2}-\mathrm{mx}_{1}+\left(\mathrm{k}_{2}+\mathrm{m}\right) \mathrm{x}_{2}=\mathrm{f}_{2}(\mathrm{t})
\end{aligned}
$$

where $f_{i}(t)=b_{i 1} u_{1}+b_{i 2} u_{2} \quad i=1,2$

Let $\quad y_{1}=\left(x_{1}, \dot{x}_{1}\right)^{T}, y_{2}=\left(x_{2}, \dot{x}_{2}\right)^{T}$,

$y=\left(y_{1}, y_{2}\right)^{T}, u=\left(u_{1}, u_{2}\right)^{T}$, the state equation is

$$
\dot{y}=A y+B u
$$

where

$$
\begin{aligned}
& A=\left[\begin{array}{cccc}
0 & 1 & 0 & 0 \\
-k_{1}-\mu & 0 & \mu & 0 \\
0 & 0 & 0 & 1 \\
\mu & 0 & k_{2}-\mu & 0
\end{array}\right] \\
& B=\left[\begin{array}{cc}
0 & 0 \\
b_{11} & b_{12} \\
0 & 0 \\
b_{21} & b_{22}
\end{array}\right]
\end{aligned}
$$

Considering the problem of wave control and letting the degree of controllability is 1 , we have 


$$
\mathrm{A}(2,2)=\left[\begin{array}{ll}
0 & 0 \\
\mu & 0
\end{array}\right] \quad \mathrm{B}(\underline{2}, 2)=\left[\begin{array}{cc}
0 & 0 \\
b_{21} & b_{22}
\end{array}\right]
$$

From criterion $1, b_{21}, b_{22}$ can not be zero simultaneously.

It can be proved that when $b_{21}=b_{22}=0$, the criterion 0 is satisfied for some $b_{11}$ and $b_{12}$. However, if the wave should be controlled on $m_{2}, b_{21}$ and $b_{22}$ must not be zero simultaneously as shown above. This is a new idea to deal with the problem of wave control.

\section{Appendix B}

Criterion 2 A structure is wave controllable, if a. $\exists \mathrm{i}(\mathrm{i}<\mathrm{n}), \mathrm{R}(\mathrm{B}(\mathrm{i}, \mathrm{r})) \supset \mathrm{A}(\mathrm{i}, \mathrm{n}-\mathrm{i}))$

b. $\exists P, \quad$ which makes rank $\left(\bar{B}, \bar{A} \bar{B}, \ldots, \bar{A}^{(n-t-1)} \bar{B}\right)=n-i$

Proof. From Criterion 1, we know the degree of controllability of Eq.(1) is i, then Eq.(a) is written as the following forms:

$$
\begin{aligned}
& \mathrm{A}_{21} \mathrm{x}(\mathrm{t}, \mathrm{n}-\mathrm{i})+\mathrm{B}_{21} \mathrm{u}_{1}+\mathrm{B}_{22} \mathrm{u}_{2}=0 \\
& \mathrm{u}_{1}=\mathrm{P}^{\mathrm{T}} \mathrm{u} \quad \mathrm{u}_{2}=\overline{\mathrm{P}}^{\mathrm{T}} \mathrm{u}
\end{aligned}
$$

Because

$$
\begin{gathered}
\mathrm{R}(\mathrm{B}(\mathrm{i}, \mathrm{r})) \supset \mathrm{R}(\mathrm{A}(\mathrm{i}, \mathrm{n}-\mathrm{i})) \\
\mathrm{R}(\mathrm{B}(\mathrm{i}, \mathrm{r}) \mathrm{P})=\mathrm{R}(\mathrm{B}(\mathrm{i}, \mathrm{r}))
\end{gathered}
$$

there exists $B_{21}{ }^{+}$, and

$$
u_{1}=-B_{21}{ }^{+}\left[B_{22} u_{2}+A_{21} x(t, n-i)\right]
$$

The state equation of $x(t, n-i)$ is,

$$
\dot{x}(t, n-i)=A_{11} x(t, n-i)+B(n-i, r) u \quad \text { i.e. }
$$$$
\dot{\mathrm{x}}(\mathrm{t}, \mathrm{n}-\mathrm{i})=\mathrm{A}_{11} \mathrm{x}(\mathrm{t}, \mathrm{n}-\mathrm{i})+\mathrm{B}_{11} \mathrm{u}_{1}+\mathrm{B}_{12} \mathrm{u}_{2}
$$

Substituting Eq.(j) into Eq.(k)

$$
\begin{gathered}
\dot{x}(t, n-i)=\left(A_{11}-B_{11} B_{21}{ }^{+} A_{21}\right) x(t, n-i) \\
+\left(B_{12}-B_{11} B_{21}{ }^{+} B_{22}\right) u_{2}
\end{gathered}
$$

The system $(\mathrm{m})$ is controllable, iff

$$
\operatorname{rank}\left(\bar{B}, \bar{A} \bar{B}, \ldots, \bar{A}^{(n-i-1)} \bar{B}\right)=n-i \text {. }
$$

So, $\exists u(t)$, which makes $\mathrm{x}(\mathrm{t}, \mathrm{i})$ wave domain controllable and $\mathrm{x}(\mathrm{t}, \mathrm{n}-\mathrm{i})$ state controllable.

\section{REFERENCES}

1. Quan Wang, Dajun Wang,"The controllability and the degree of controllability in wave control of structure", CHINESE SCIENCE BULLITIN, 1994, Vol.29, N0.4, pp. 307-308

2. Quan Wang, Dajun Wang, "Some researches and new concepts about the controllability of structural wave control", ISTS'94-s-10, International Symposium on
Space Technology and Science, Yokoham, May 15-24, 1994

3. Quan Wang, Dajun Wang and Xianyue Su, "Researches of wave domain control and a reduced order model of structure", Scientia Sinica, 1994, Vol.24, No.9, pp. 1216-1222

4. Quan Wang, Dajun Wang, "A reduced order model about structural wave control based upon the concept of degree of controllability", IEEE Trans. on Automatic Control, 1994, Vol.39, No.9, pp. 1711-1713

5. Quan Wang, Dajun Wang, "Some methods and problems in structural wave control", Asia and pacific Conference on Aerospace Technology and Science, Hangzhou,Oct. 1994

6. Hughes, P. C., Skelton,R.E.,"Modal truncation for flexible spacecraft", J. of Guidance and Control, 1981, Vol.4, No.3, pp. 291-297

7. Gibson, J. S., "A note on stabilization of infinite dimensional linear oscillators by compact linear feedback", SIAM J. on Control and Optimization, 1986, Vol.18, No.3, pp. 311-316

8. Balas, M. J., "Finite-dimentional control of distributed parameter systems by galerkin approximation of infinite dimensional controllers", J. of Mathematical Analysis and Applications, 1986, Vol.114, pp. 17-36

9. Skelton, R. E., "Observability measures and performance sensitivity in the model reduction problem", International J. Control, 1979, Vol.29, No.4, pp. 541-556

10. Sezer, M. E., Siljak, D. D., "Validation of reducedorder models for control system design", J, of Guidance and Control, 1982, Vol.5, No.5, pp. 430-437

11. Skelton, R. E., Hughes,P.C.,"Order reduction for models of space structures using modal cost analysis", J. of Guidance and Control, 1982, Vol.5, No.4, pp. 351357

12. Meirovitch, L., Oz, H., "Modal-space control of distributed gyroscopic systems", J. of Guidance and Control, 1980, Vol.3, No.2, pp. 140-150

13. Sesak, J. R., Likins, P., "Flexible spacecraft control by model error sensitivity suppression", J. of Astronautical Sciences, 1979, Vol.27, No.2, pp. 131156

14. Hale, A. L., Rahn, "Robust control of self-adjoint distributed-parameter structures", J. of Guidance, Control and Dynamics, 1984, Vol.7, No.3, pp. 265-273

15. Rogers, R. O., Sworder, D. D., "Suboptimal control of linear systems derived from models of lower dimension", AIAA J., 1971, Vol.9, No.8, pp. 14611467 


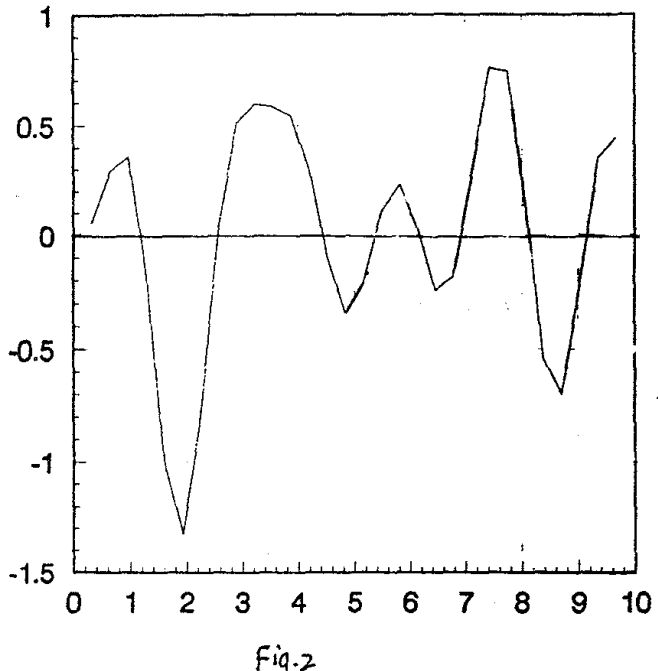

Fig. 2
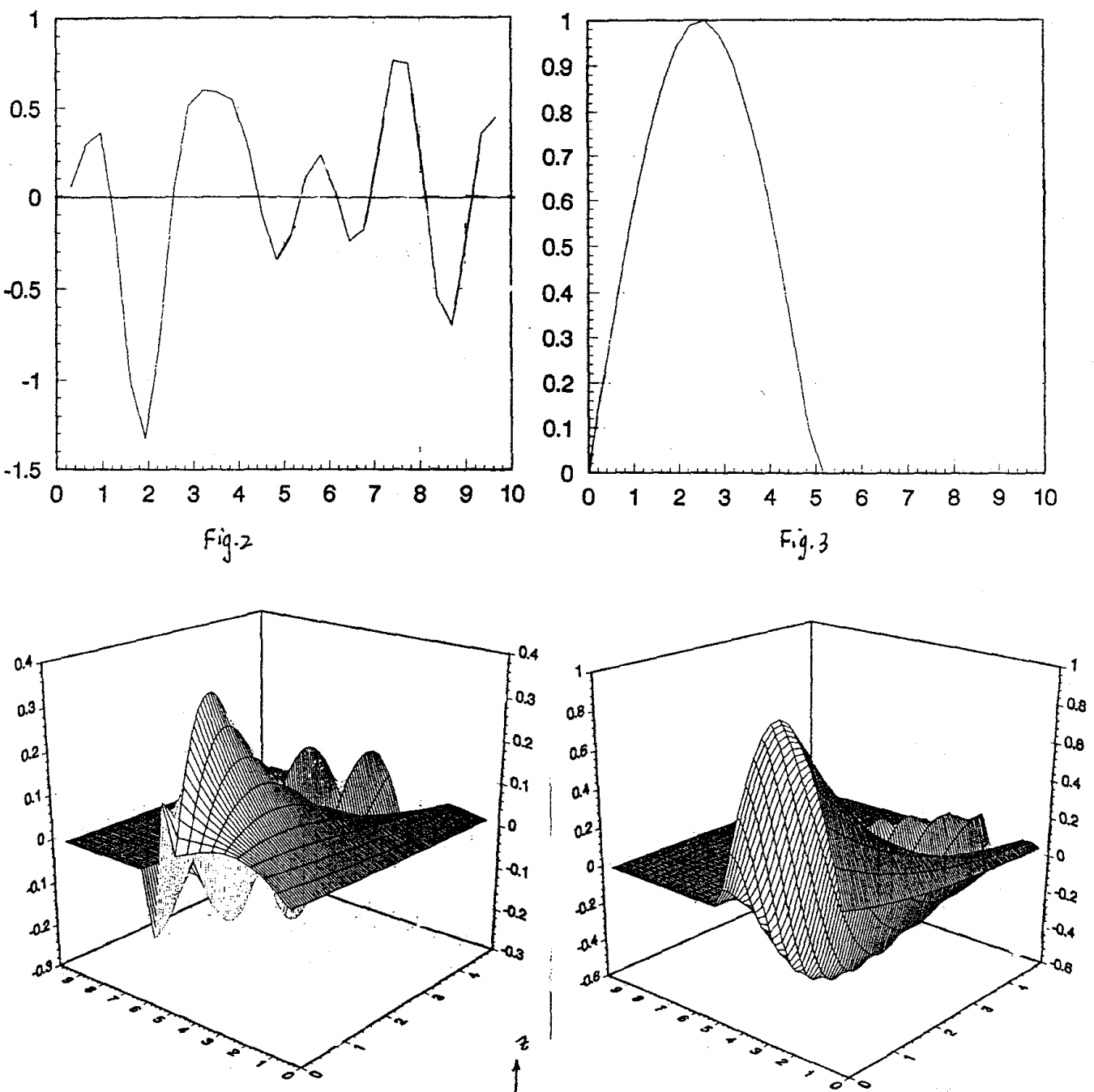

Fig. 4

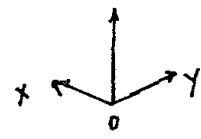

Fig. 5

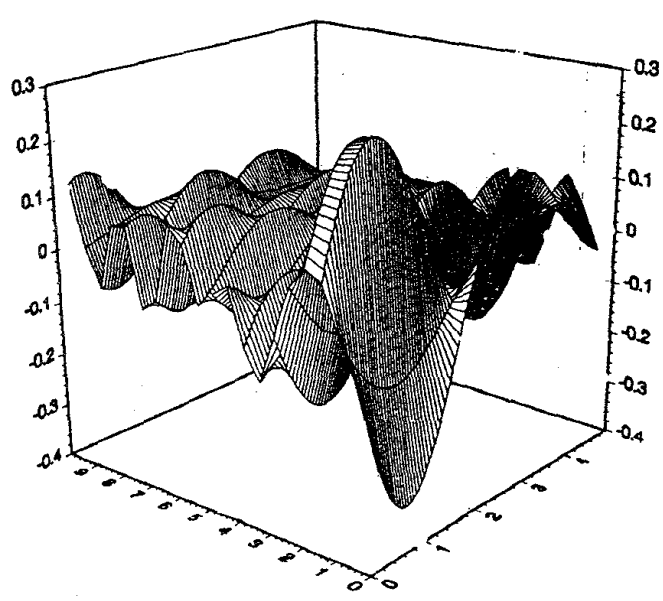

Fig. 6

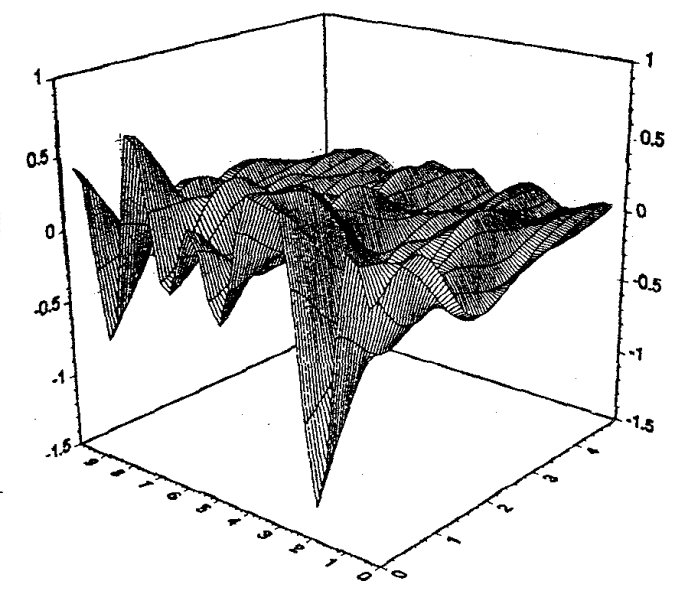

Fig.? 